\title{
grupo escolar Le Pontet
}

ROGER ADATTE, arquitecto

STUCKY-PANCHAUD-DERRON, ingeniero

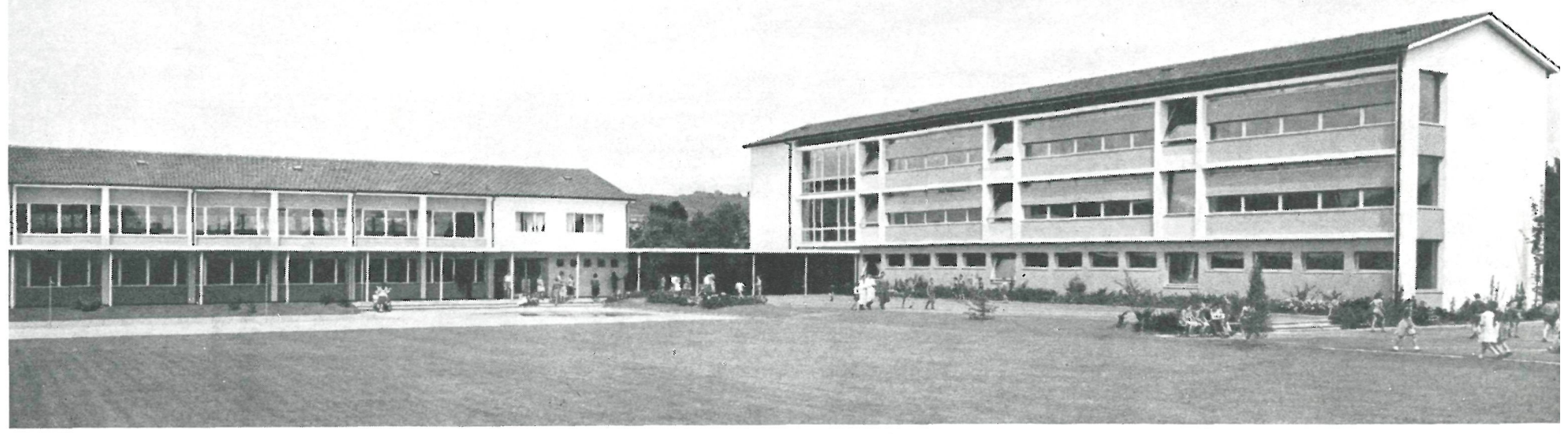

$141 \cdot 52$
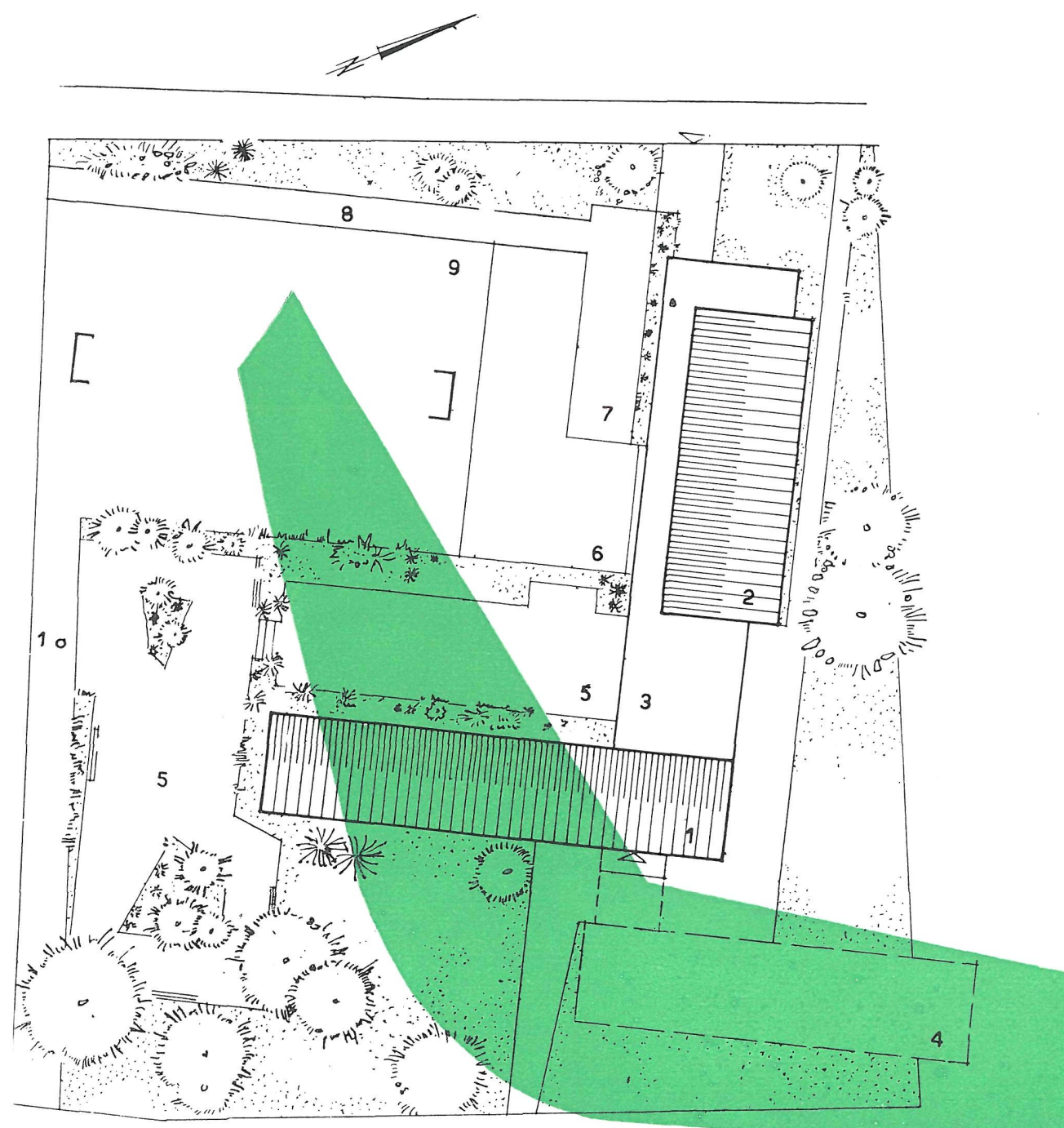

Un importante grupo escolar se ha construído en los arrabales de esa industriosa ciudad que se llama Lausanne, cuyo veloz crecimiento da pie a necesidades nuevas, y cuyas exigencias sólo pueden ser cubiertas por hombres cultos, educados, estudiosos, aptos para el feliz ejercicio de su inteligencia. La enseñanza oficial no es-no debe ser-un privilegio de minorías, y las autoridades municipales de Ecublens-entendiéndolo así-abrieron en el año 1954 libre concurso para la edificación de un grupo escolar que acogiera a sus más jóvenes vecinos y que los pusiera en condiciones de continuar la transformación industrial-ciudadana, europea, universal; así lo exige la humana inter-relación-por sus mayores comenzada.

\section{situación}

1. Colegio.-2. Gimnasio--3. Patio cubierto y garaje de bicicletas - 4. Ampliación, segunda etapa.-5. Patio.-6. Zona de juego.-7. Foso de arena.-8. Pista de saltos.-9. Juegos. Campo de deportes. 10. Pista de carreras. 


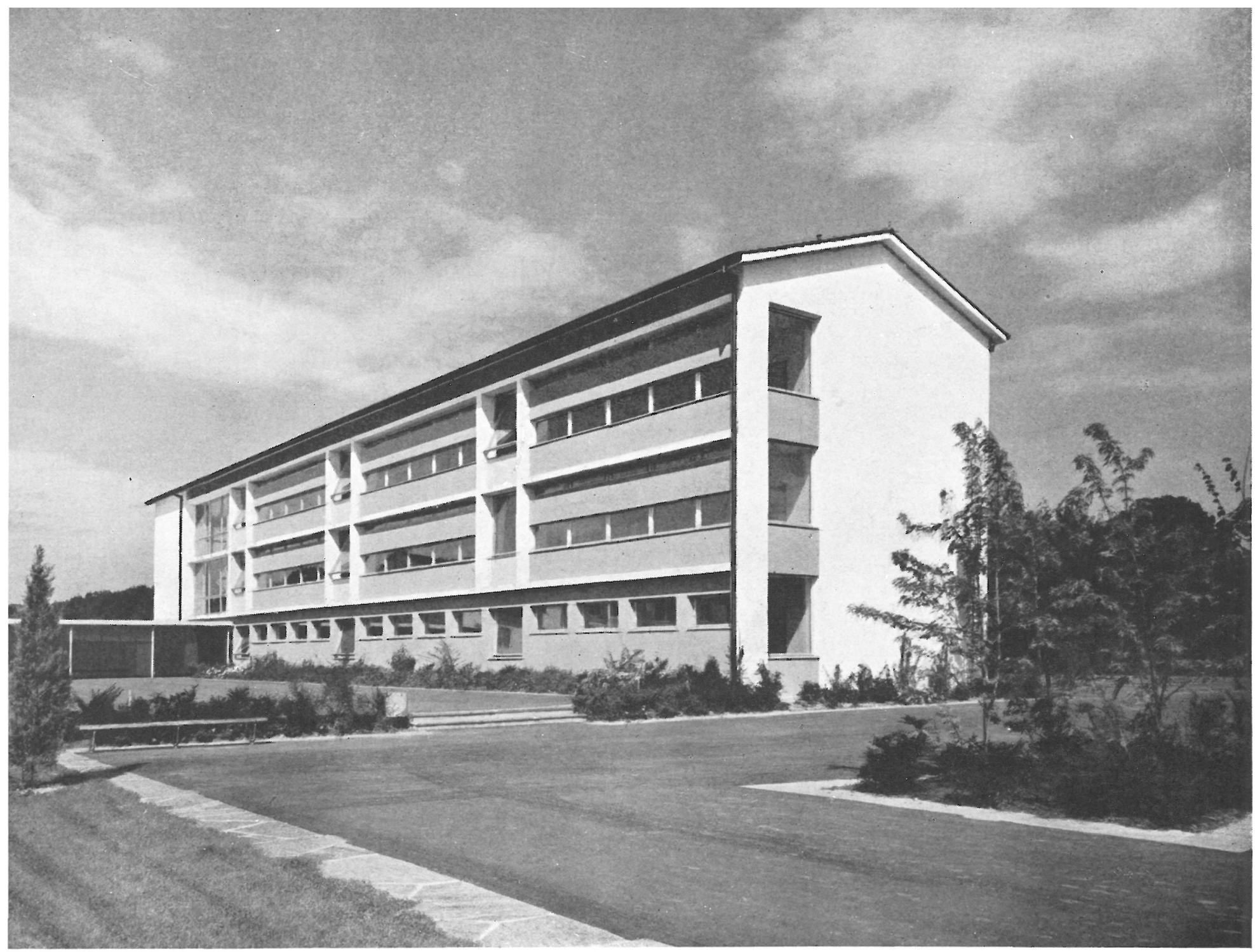

Tras las deliberaciones pertinentes, el trabajo fué encomendado al arquitecto señor Adatte, en gracia a los merecimientos del proyecto que en su día presentó.

Se eligió-en primer lugar y recordando siempre la enorme influencia del medio ambiente en el individuo-un lugar despejado, anchuroso, de fácil acceso, sin contacto con las vías urbanas de tráfico intenso, con visión frontal del paisaje alpino, y rodeado por zonas verdes, donde los cerebros infantiles pudieran olvidar sus faltas de ortografía y las caras severas de sus profesores. Ya nadie admite--ni quiere, ni cree-aquello de que «la letra con sangre entra». La letra entra con alegría, con esperanza y con sencillez. Vayan, pues, pinos y manantiales, paredes muy blancas y ventanas más abiertas, bicicletas gentiles y monigotes danzando su simpática incoherencia en las pizarras. Las aulas miran al este y al sur, a oriente y mediodía, para que el sol mañanero entre, despeje corazones, aclare ideas e inspire optimismo. También el deporte tiene su lugar. Nadie-tampoco-olvida que el cuerpo es soporte e instrumento, necesidad y herramienta de la cultura. Un moderno gimnasio--provisto de cuanto el más exigente atleta pueda soñar- se levanta cercano al edificio principal, pero lo suficientemente alejado para no perturbar, con ruidos, tentaciones y sugerencias, la vida intelectual de los alumnos y el desarrollo eficaz de las lecciones. 


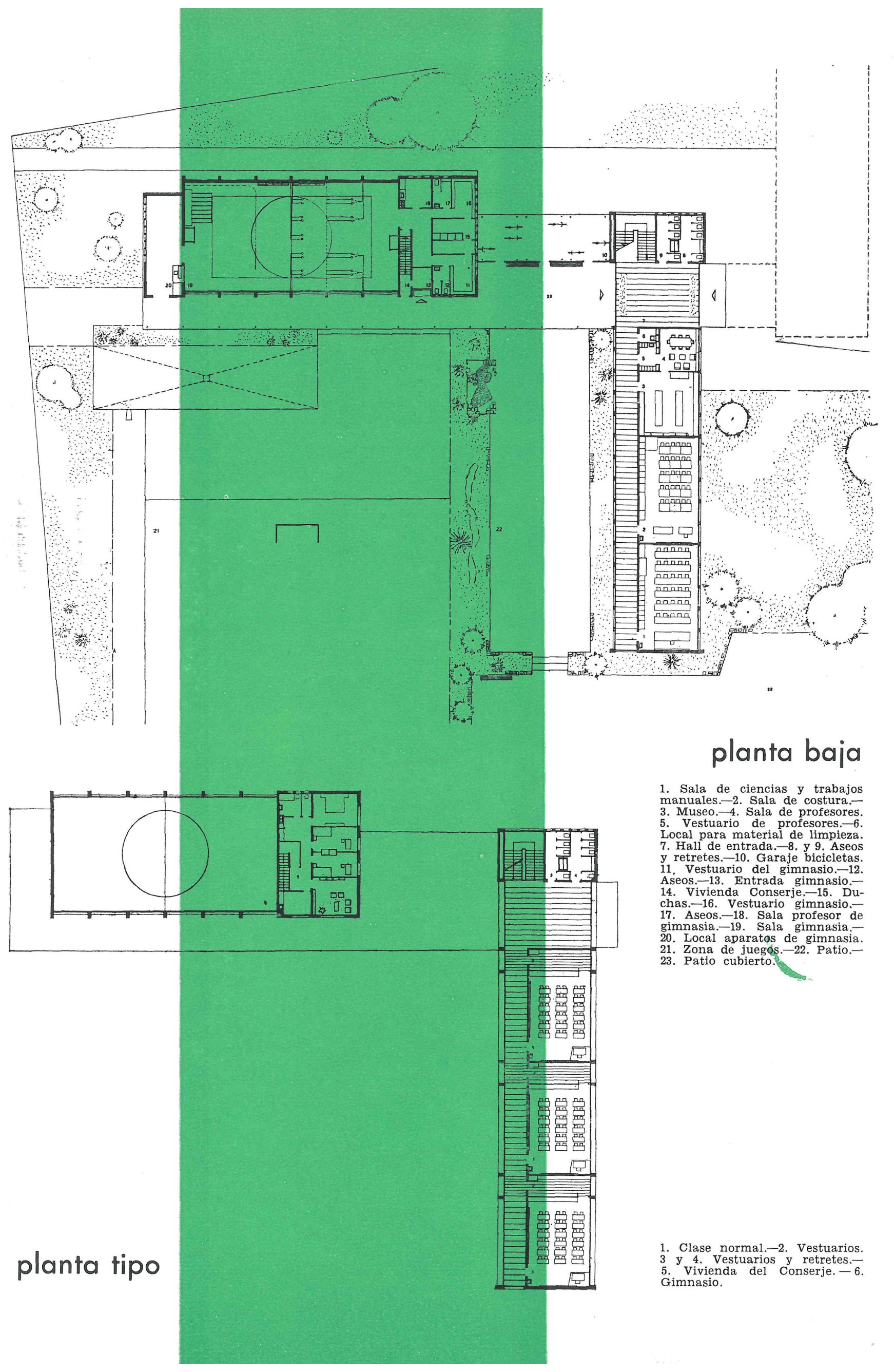



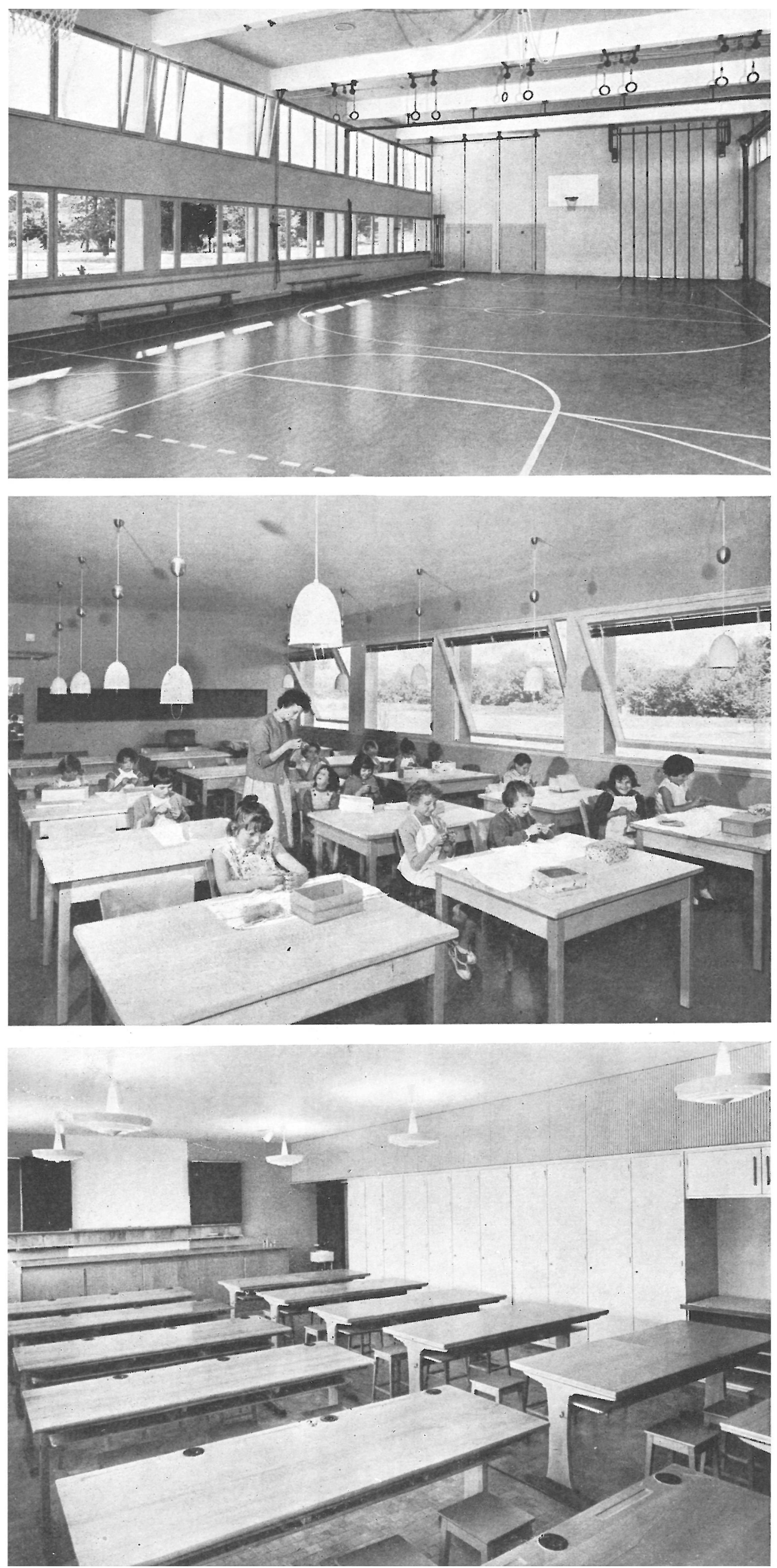

Los habitantes adultos de Ecublens-miembros de las sociedades deportivas locales-tienen acceso directo desde el exterior, y pueden allí estirar sus músculos, rejuvenecer su concepción del mundo, y engrasar las bisagras de su buen humor.

Hemos dicho que la cultura no entra por las bravas. Hemos dicho que el cultivo de la inteligencia requiere un paralelo cultivo de las facultades corporales. Añadamos todavía-sin pretensiones de ninguna clase-otro teorema pedagógico de amplia validez: teoría y práctica son hermanas siamesas. Pensando en ello-icómo no!-los autores del grupo escolar que hoy explica su lección en estas páginas, han dispuesto laboratorios físicos y químicos, talleres de trabajo manual, pequeños museos de ciencias naturales y salas para la proyección de películas que, enseñando, diviertan; divirtiendo, enseñen.

A continuación se adjuntan planos y leyendas que analizan los edificios en cuestión de una manera científica. Pero existen cosas que no pueden descubrirse en el academicismo correcto, en la fría inhospitalidad del lenguaje técnico. Cosas como las que ya hemos dicho. Cosas como éstas--muy pocas yaque vamos a decir:

En cada aula 36 alumnos estudian; un profesor-elevado sobre renacentista podio-explica, y una pizarra de seis movibles brazos se entrega generosa a las operaciones aritméticas, a las declinaciones latinas.

En cada aula hay-también-un altavoz que trae las emisiones radiofónicas a la escuela.

En cada aula existe un vestuario de armarios bajos y cristales superiores, perfectamente controlable por el profesor.

En cada aula mueren los sonidos del exterior, ejecutados por alertas centinelas-paredes absorbentes de sonidos.

En cada aula los colores son claros y escogidos, llamada a mentes claras y escogidas.

En cada aula... ¡Quién pudiera estudiar en ellas! 